\title{
Comprehensive rural epilepsy surveillance programme in Uttarakhand state of India
}

Sir,

Uttarakhand is the youngest state of India covering hills of Himalayas. We conducted community-based crosssectional study to determine the prevalence and profile 
of seizures disorders in Chakrata block of Dehradun district in Uttarakhand state. This block is having lowest composite block index (CBI) and is about 80 kilometers form Dehradun and is 6000 to 7000 feet above the sea level. As epilepsy has an expected prevalence of about 5 to 10 per 1000 , a sample size of about 10,000 is felt to be adequate to determine the prevalence in the community. Thirtysix villages from all 9 nyaya panchayat were randomly selected to cover $25 \%$ population. Field staff was trained for a week in the survey techniques and data recording by door-to-door survey. All positive responders for epilepsy were ascertained by a neurologist to find cases with definite epilepsy and all of them had contrast computed tomography (CT) of brain and electroencephalography (EEG) to find the possible etiology. Various demographic parameters collected were compared among cases and randomly selected age matched controls.

There were 1671 households with a population of 14086 including 3991 (28.3\%) children. The gender distribution was: Male 7466 (53\%) and female 6620 $(47 \%)$. Main occupation of the population was farming (91\%) and the average monthly income was INR 1957 per household. Only $11.3 \%$ houses had in-built toilet facility.

Of the 14086 people surveyed, there were 141 people with two or more seizures giving a prevalence of 10 per 1000 seizure disorders in this population. This population included both acute symptomatic (provoked) seizures and also unprovoked seizures. The prevalence of unprovoked seizures, epilepsy (two or more seizures) was 7.5 per 1000 population. The prevalence of febrile seizures was 2.27 per 1000 population (32 children aged less than 7 years).

The most common seizure type was generalized tonic-clonic seizures in 76 (53.9\%). Only 41 (29\%) had ever used allopathic medicine to control the seizures. Of the 141 people with seizure disorder, $138(95.1 \%)$ patients had active disease. Prevalence of non-fatal head-injury with at least 24 hours loss of consciousness in this society was 3.98 per 1000 ( 56 cases). Relative risk of seizures was higher with febrile convulsion (odd ratio - infinite), family history of seizures (odd ratio - infinite) and head injury (odd ratio-3.74 and CI - 1.34-10.46). The distribution of families with more than six members living in a single room was similar between the families with seizure disorder and the control population, $24(17.7 \%)$ vs. 25 $(18.4 \%)(P=0.48)$. Factors like animal contact, dietary pattern, hand-washing habits, immunization practices, in-house toilet, mental retardation and history febrile encephalopathy were not found to be significant risk for seizures.

Our study reflects the prevalence of seizure (provoked and unprovoked) disorders in this community. Neurocysticercosis was the putative risk factor for seizures in 49 (34.8\%), active lesions in 35 and calcified lesions in 14 patients. The next common putative risk factor was gliotic lesion related to traumatic brain injury and stroke in $22(15.6 \%)$ patients. Eleven $(7.8 \%)$ patients had idiopathic generalized epilepsy. In the remaining 59 (41.8\%) patients no cause could be identified. The prevalence of seizure disorder related to neurocysticercosis was 2.5 per 1000 .

The prevalence of epilepsy in this population was higher than the reported prevalence in the metaanalysis of all the community-based studies in India. ${ }^{[1]}$ In our study sex specific prevalence showed reverse ratio with higher in females (5.8) than male (4.2 per 1000) when compared with the National Figures of sex-specific prevalence for men and women are 5.25 and 4.56 respectively. ${ }^{[1]}$ Treatment gap in our study like others was $71 \%$. The prevalence of seizure disorder related to neurocysticercosis was higher than the reported prevalence from Vellore. ${ }^{[2]}$

\section{Acknowledgment}

This project was supported by Indian Council Medical Research (CMR). We thank for all the needful provided by ICMR during this study.

\section{Deepak Goel ${ }^{1}$, Alka Agarwal*, J S Dhanai*,} V.D.Semval ${ }^{2}$, V. Mehrotra ${ }^{2}$, V. Saxena ${ }^{2}$, B. Maithili ${ }^{3}$

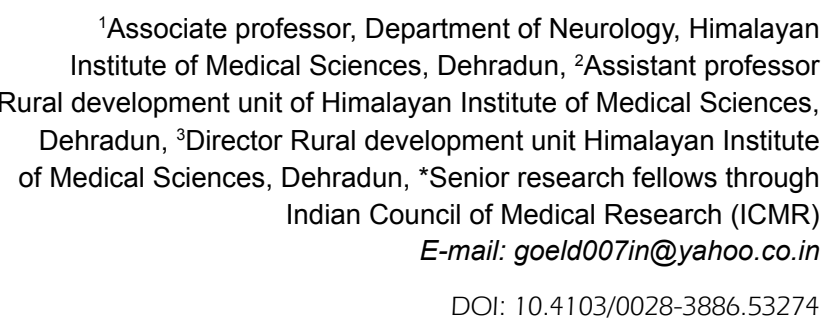

\section{References}

1. Sridharan R, Murthy BN. Prevalance and pattern of epilepsy in India. Epilepsia 1999; 40:631-6.

2. Rajshekhar V, Venkat Raghava M, Prabhakaran V, Oommen A, Muliyil AJ. Active epilepsy as an index of burden of neurocysticercosis in Vellore district, India. Neurology 2006;67:2135-2139.

Accepted on 04-06-2009 